\title{
Spectral tuning and near-field imaging of photonic crystal microcavities
}

\author{
Francesca Intonti, ${ }^{1,2}$ Silvia Vignolini, ${ }^{1}$ Francesco Riboli, ${ }^{1}$ Anna Vinattieri, ${ }^{1}$ Diederik S. Wiersma, ${ }^{1}$ Marcello Colocci, ${ }^{1}$ \\ Laurent Balet, ${ }^{3}$ Christelle Monat, ${ }^{3}$ Carl Zinoni, ${ }^{3}$ Lianhe H. Li, ${ }^{3}$ Romuald Houdré, ${ }^{3}$ Marco Francardi, ${ }^{4}$ \\ Annamaria Gerardino, ${ }^{4}$ Andrea Fiore, ${ }^{3}$ and Massimo Gurioli ${ }^{1}$ \\ ${ }^{1}$ LENS and Department of Physics, University of Florence, 50019 Sesto Fiorentino, Italy \\ ${ }^{2}$ CNISM, Unità di Ricerca di Firenze, 50019 Sesto Fiorentino, Italy \\ ${ }^{3}$ Ecole Polytechnique Federale de Lausanne, Institute of Photonics and Quantum Electronics, CH-1015 Lausanne, Switzerland \\ ${ }^{4}$ Institute of Photonics and Nanotechnology, CNR, 00156 Roma, Italy
}

(Received 8 May 2008; published 1 July 2008)

\begin{abstract}
We experimentally observe a sizable and reversible spectral tuning of the resonances of a two-dimensional photonic crystal microcavity induced by the introduction of a subwavelength size glass tip. The comparison between experimental near-field data, collected with $\lambda / 6$ spatial resolution, and results of numerical calculations shows that the spectral shift induced by the tip is proportional to the local electric field intensity of the cavity mode. This observation proves that the electromagnetic local density of states in a microcavity can be directly measured by mapping the tip-induced spectral shift with a scanning near-field optical microscope.
\end{abstract}

DOI: 10.1103/PhysRevB.78.041401

PACS number(s): 42.70.Qs, 68.37.Uv, 78.67.Hc

Photonic crystals are periodic dielectric structures in which light flow is forbidden at certain frequencies and along given directions. ${ }^{1}$ By tailoring the design of the periodic structure, one can control the light propagation and can engineer the local density of states of the electromagnetic field. Among the several fascinating applications of photonic crystals, the possibility to introduce localized dielectric defects leads to the formation of states into the forbidden energy gap corresponding to spatially localized optical modes. These structures are often denominated photonic crystal microcavities (PCMCs) and with appropriate design allow to realize optical modes with quality factor $(Q)$ larger than $10^{6} .^{2}$ Such microcavities have been already successfully exploited for the realization of low-threshold lasers, ${ }^{3,4}$ single photon sources,${ }^{5}$ add-drop filters, ${ }^{6}$ and for the implementation of cavity quantum electrodynamics experiments. ${ }^{7-9}$ Crucial issues in the progress of PCMCs are the possibility to control, to tune, and to carefully probe the optical modes.

In this paper we report on to two important achievements: (i) the high fidelity determination of the optical local density of states (LDOS) and of the tip-induced losses in PCMCs by scanning near-field optical microscopy (SNOM) and (ii) the reversible tuning of the PCMC resonance frequencies. Tuning has been attempted so far with various methods, including temperature sensitivity, ${ }^{8}$ digital etching, ${ }^{9,10}$ liquid crystal infiltration, ${ }^{11,12}$ adsorption of gas molecules, ${ }^{13,14}$ and nano-oxidation. ${ }^{15}$ Most of these techniques either modify the properties of the whole sample, avoiding the selective tuning of a single microcavity, or the tuning is achieved in an irreversible way. A very exciting possibility to tune microcavity resonances has been proposed by Koenderink et al., ${ }^{16}$ using the glass tip of a SNOM apparatus. Initial experimental studies show that the transmission spectra of photonic crystal waveguides with integrated PCMC can indeed be modified. ${ }^{17-20} \mathrm{We}$ experimentally demonstrate that the approach presented in Ref. 16 is indeed feasible. The local introduction of a subwavelength dielectric $\mathrm{SiO}_{2}$ tip can be used for accurate tuning of the resonance frequency of a photonic crystal microcavity without necessarily introducing significant losses.
While performing these studies, we found that the same principle can be used as a powerful method for mapping the electromagnetic LDOS, which is essential for the proper characterization of photonic structure in general. Even if SNOM experiments have proven to have enough sensitivity and spatial resolution for this purpose, ${ }^{21-23}$ still an unambiguous and artifact free determination of the LDOS has not been achieved so far, partly due to the complex interaction between SNOM tip and sample. We show in this paper that a very good measure for the local electric field intensity is the tip-induced spectral shift. By constructing maps of this shift one obtains an imaging method which reproduces the LDOS with much higher fidelity then conventional SNOM methods. In addition, maps of the broadening of the resonance peaks can be used to determine the tip-induced losses and obtain important insights on the SNOM detection mechanisms.

The investigated two-dimensional (2D) photonic crystal microcavity incorporates quantum dots (QDs) that act as local light source and is fabricated on a suspended membrane to ensure a confinement in the vertical direction by total internal reflection. We use a GaAs based heterostructure: three layers of high-density InAs QDs emitting at $1300 \mathrm{~nm}$ are grown by molecular beam epitaxy at the center of a 320-nm-thick GaAs membrane. ${ }^{24}$ The structure under consideration consists of a 2D triangular lattice of air holes with lattice parameter $a=301 \mathrm{~nm}$ and filling fraction $f=35 \%$, where the cavity is formed by four missing holes organized in a diamondlike geometry [Fig. 1(a)]. The first two resonances of this cavity are characterized by orthogonal polarizations, i.e., the spatial distribution of the modes appears elongated in perpendicular directions. For imaging purposes, pronounced differences are helpful to unambiguously identify meaningful features and to directly compare experimental data with calculated results. A commercial SNOM (Twinsnom, OMICRON) is used in an illumination-collection geometry with a spectral resolution of $0.1 \mathrm{~nm}$. In this geometry, the sample is excited with light from a diode laser $(780 \mathrm{~nm})$ coupled into the tip that is raster scanned at a constant height, smaller than $5 \mathrm{~nm}$, on the sample surface. Following the proposal of Ref. 16, we use a pure dielectric tip as 
(a)

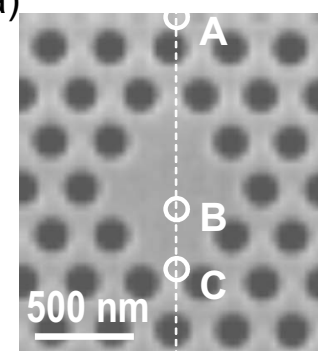

(b)

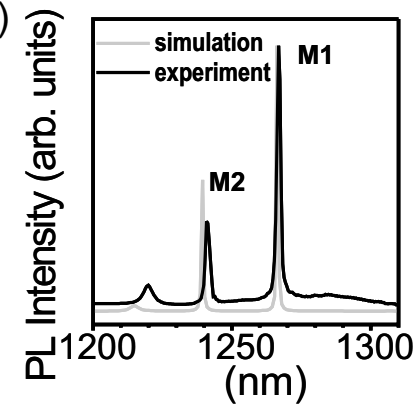

(c)

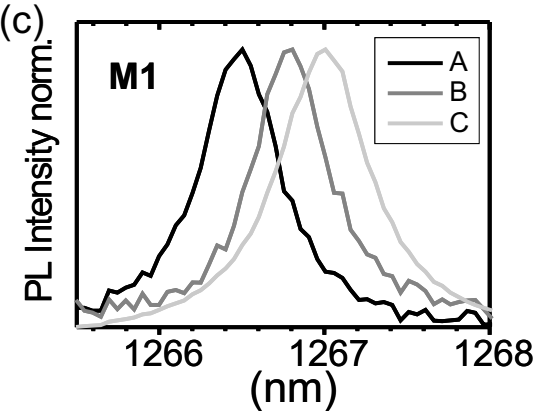

FIG. 1. (a) Scanning electron microscopy (SEM) image of the investigated sample. The three white circles indicate the position of the tip where the spectra of (c) were collected. The dashed line indicates the sample region considered in Fig. 3(b) Experimental (black line) and calculated (gray line) PL spectra. (c) PL spectra collected at three different tip positions as indicated in (a).

a chemically etched uncoated near-field fiber probe. ${ }^{25}$ The use of a coated tip would not significantly improve the spatial resolution, but would have dramatic consequences in the perturbation induced by the tip with consequent complications in the interpretation of the measurements. Photoluminescence (PL) spectra from the sample were collected at each tip position through the same probe and the PL dispersed by a spectrometer was detected by a cooled InGaAs array. The overall spatial resolution, obtained from the experimental data (as described later) is of the order of $200 \mathrm{~nm}$, which is small enough to observe the details of the optical LDOS of the PCMC and, at the same time, large enough to have a good signal to noise ratio in the PL measurements. In order to have theoretical simulations of the LDOS in our PCMCs, numerical calculations were performed with a commercial three-dimensional (3D) finite-difference timedomain (FDTD) code (CrystalWave, Photond). Computational meshes were 15 grid points per period.

The experiment has been performed under excitation conditions that determine the population of both the ground and first excited states of the embedded QDs, therefore we observed emission in the wavelength range between $1190 \mathrm{~nm}$ and $1310 \mathrm{~nm}$. The black curve of Fig. 1(b) shows a typical emission spectrum of the structure under investigation characterized by two main peaks, $M 1$ and $M 2$, centered around $1266.8 \mathrm{~nm}$ and $1241.3 \mathrm{~nm}$, respectively. In particular, the resonance at longer wavelengths is characterized by a full width at half maximum (FWHM) of $0.5 \mathrm{~nm}$, corresponding to a quality factor larger than 2400. In the same graph, the gray line represents the calculated spectrum obtained in our 3D FDTD calculation, that reproduces the exact geometry of the sample, assuming a GaAs refractive index of 3.46. The agreement between the experimental and theoretical curves is very good. From the calculation we extract for $M 1$ a $Q$ of the order of 4500. The slightly smaller experimentally observed $Q$ can be reasonably attributed to the disorder (i.e., surface roughness, fluctuations in the pore size, and not perfect cylindrical shape of the pores) introduced during the processing of the real structure. Figure 1(c) reports three normalized PL spectra of the $M 1$ resonance recorded with the tip located at three different sites of the sample along its vertical axis, as indicated in Fig. 1(a). The spectrum recorded at the apex of the microcavity [position $C$ in Fig. 1(a)] shows a clear redshift with respect to the spectrum recorded outside the microcavity [position $A$ in Fig. 1(a)].

From all the spectra collected during a SNOM scan covering an area equal to $1.40 \times 1.15 \mu \mathrm{m}^{2}$ [Fig. 2(a)], it is possible to extract the 2D spatial map of the spectral shift for the mode $M 1$, as shown in Fig. 2(b). The map, in a blue-to-white color scale, was obtained by fitting with a Lorentzian curve every emission spectrum in the wavelength range $1260-1275 \mathrm{~nm}$, and taking from every fitted curve the central wavelength of the cavity resonance as a function of the tip position. Following the predictions presented in Ref. 16, that demonstrate a direct correspondence between the frequency shift and the unperturbed mode profile, we report in Fig. 2(c) the spatial distribution of the electric field intensity $\left(E^{2}\right)$ associated with the mode $M 1$, obtained with 3D FDTD
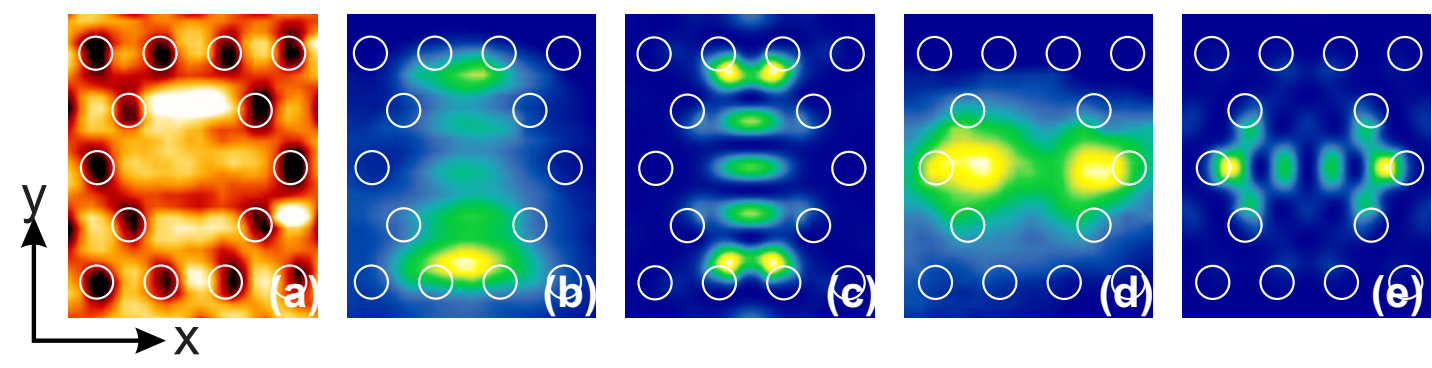

FIG. 2. (Color online) (a) Sample topography acquired concurrently with the optical data. (b) Map of the tip-induced spectral shift of resonance $M 1$, maximum redshift $=0.5 \mathrm{~nm}$. (c) Calculated spatial distribution of $E^{2}$ related to mode $M 1$. (d) Map of the tip-induced spectral shift of resonance $M 2$, maximum redshift $=0.4 \mathrm{~nm}$. (e) Calculated spatial distribution of $E^{2}$ related to mode $M 2$. The position of the photonic crystal pores is superposed to guide the eye. All the images cover an area of $1.15 \times 1.40 \mu \mathrm{m}^{2}$ and except (a) are plotted on a blue-to-white color scale (grayscale). 

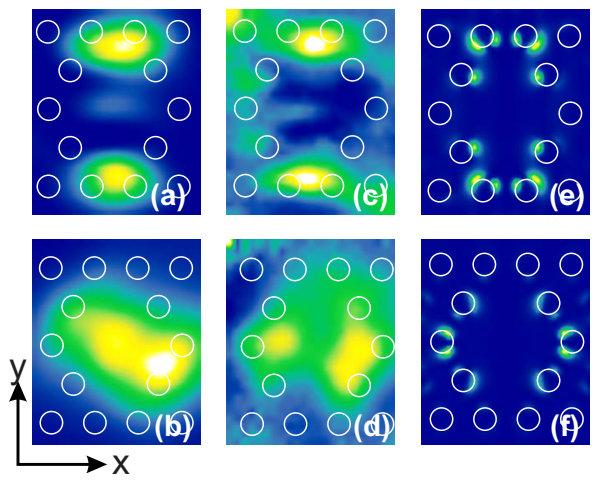

FIG. 3. (Color online) Spatial distribution of the PL signal related to resonance $M 1$, integrated in the wavelength range between $1266.2 \mathrm{~nm}$ and $1267.2 \mathrm{~nm}$. (b) Spatial distribution of the PL signal related to resonance $M 2$, integrated in the wavelength range between $1240.2 \mathrm{~nm}$ and $1241.2 \mathrm{~nm}$. (c) Map of the spectral broadening of resonance $M 1$; minimum broadening $=0.52 \mathrm{~nm}$ and maximum broadening $=0.65 \mathrm{~nm}$. (d) Map of the spectral broadening of resonance $M 2$; minimum broadening $=0.56 \mathrm{~nm}$ and maximum broadening $=0.75 \mathrm{~nm}$. (e) Calculated spatial distribution of $E_{z}^{2}$ related to mode $M 1$. (f) Calculated spatial distribution of $E_{z}^{2}$ related to mode $M 2$. The position of the photonic crystal pores is superposed to guide the eye. All the images cover an area of 1.15 $\times 1.40 \mu \mathrm{m}^{2}$ and are plotted on a blue-to-white color scale (grayscale).

simulations. The comparison between the experimental data and the numerical results clearly indicates that the map of the spectral shift directly corresponds to the electric field intensity associated with the resonance. The spatial resolution, defined as the full width at half maximum of the profile of the smallest feature that can be resolved, extracted from Fig. 2(b) is $200 \mathrm{~nm}$. The same analysis performed on the mode M2 show analogous results. Figure 2(d) and Fig. 2(e) report the map of the spectral shift and the spatial distribution of $E^{2}$ associated with the mode $M 2$, respectively. These results show that the theoretical prediction of a mode perpendicular polarized to $M 1$ is well reproduced by the map of the spectral shift extracted from the experimental data in the wavelength range $1235-1250 \mathrm{~nm}$. Measurements of the spectral shift therefore form an ideal method for characterizing the modes of the system. In conventional SNOM experiments the PL intensity is assumed to directly correspond to the spatial distribution of the electric field intensity. ${ }^{21,22}$ The results shown in Figs. 3(a) and 3(b), which report measurements of the intensity, demonstrate that this conventional method leads to less detailed maps and worse spatial resolution $(250 \mathrm{~nm})$. The map of the spectral shift represents a cleaner measurement of the LDOS with respect to the map of the PL intensity. This is a very important result, which is related to the fact that the PL intensity collected through the tip can be affected by different parameters, as the not perfect coincidence of the physical shape of the tip and its optically active region, the eventuality that the tip collects a mixture of evanescent and propagating waves, and the possible inhomogeneity of the quantum dots. Since the strength of the tipinduced spectral shift is proportional to the electric field intensity of the eigenmode, ${ }^{16}$ this improved imaging method

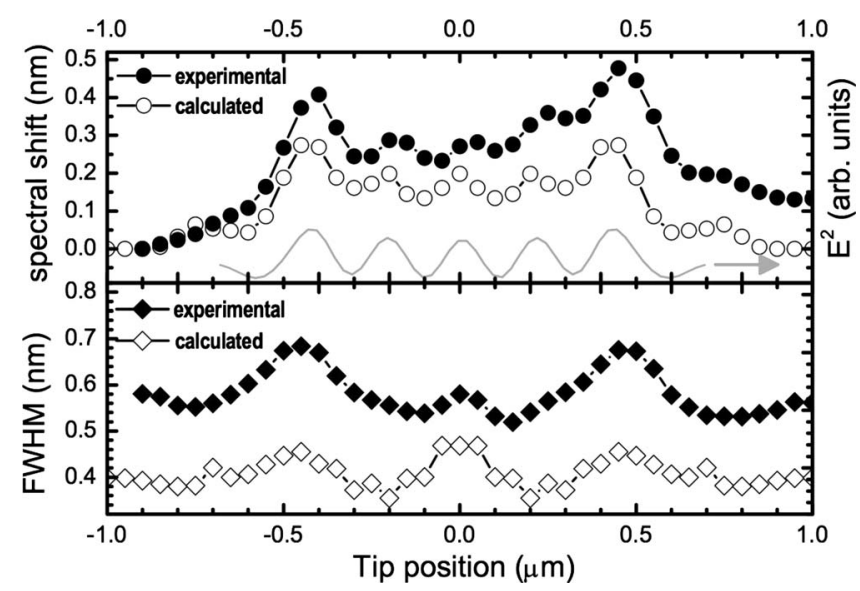

FIG. 4. Upper panel: Experimental (filled circles) and calculated (open circles) tip-induced spectral shift of the $M 1$ resonance. The gray line reports the vertical cross section, $200 \mathrm{~nm}$ wide, in the middle of the calculated electric field intensity reported in Fig. 2(c). Lower panel: Experimental (filled diamond) and calculated (open diamond) tip-induced spectral broadening of the $M 1$ resonance.

can be generalized to any microcavity design.

An exciting option of the tip induced tuning is the possibility to perturb the optical mode without introducing additional losses. Figure 3(c) and Fig. 3(d) show the map of the resonance broadening of the modes $M 1$ and $M 2$, respectively. The images are obtained by reporting in a blue-towhite color scale the values of the FWHM extracted by the same Lorentzian fits performed to create the maps of the spectral shift [see Fig. 2(b) and Fig 2(d)]. Numerical calculations ${ }^{16,26}$ already clarified that, even though the losses are expected to be larger where the perturbation of the tip is larger, the relation between the spectral shift and the induced losses is nontrivial, due the fact the scattering into the tip is sensitive to the polarization of the mode at the tip position, i.e., is much more efficient for tip positions where the electric field has a large $z$ component. ${ }^{27}$ Our experimental data show that there are positions where the tip-induced tuning presents a local maximum and the spectral broadening has a local minimum, as, for instance, for the resonance $M 1$ in the regions just above and below the center of the cavity. This result is in qualitative agreement with the spatial distribution of $E_{z}^{2}$ related to $M 1$ [Fig. 3(e)], that, differently from $E^{2}$ [Fig. 2(c)], shows large intensity only in the regions around the vertical diamond apex, and an intensity minimum in the central area of the cavity. It is also extremely interesting that the maps of the resonance broadening exhibit a close resemblance to the intensity maps: the SNOM tip induces more losses at the locations where it collects more signal. This also demonstrates that the SNOM PL intensity cannot quantitatively reproduce the local density of states.

In order to gain more quantitative understanding, Fig. 4 shows an analysis of the spectral position and FWHM of the M1 mode. The experimental data of Fig. 4 are obtained by extracting the line profiles along the $y$ direction in the middle of Fig. 2(b) and Fig. 3(c). The peak wavelength together with the FWHM is reported in Fig. 4 as a function of the tip position along the dashed line of Fig. 1(a). The measured values are compared with the results of 3D FDTD calcula- 
tions. The geometry is composed by a dielectric cylindrical tip, refractive index 1.6 and diameter $200 \mathrm{~nm}$, in contact with the PCMC. In the upper part of Fig. 4 is also reported a vertical cross section of the calculated distribution of the electric field intensity collected along the $y$ direction in the middle of the image Fig. 2(c). The agreement between experiment and theory is remarkable. The behavior of the peak spectral position (circles) and of the electric field intensity (gray line) confirms that the tip-induced frequency shift follows the $E^{2}$ spatial profile. At the same time, also the FWHM of the cavity resonance (diamonds) has a well defined profile, associated to the cavity losses induced by the tip. From the data reported in Fig. 4 we find that the maximum tipinduced mode tuning, $0.5 \mathrm{~nm}$, is as large as the resonance itself. It means that we observe a $100 \%$ tuning of the resonance peak in FWHM units and correspondingly the $Q$ is reduced only about $20 \%$. More generally the spectral shift is accompanied by small changes (even negligible in specific tip positions) in the quality factor indicating that the photonic properties of the device are only slightly perturbed.

Our results open approaches to finely correct and dynamically tune the resonant modes of photonic crystal structures. The flexibility of the presented technique can be exploited for achieving the spectral overlap between resonant modes and embedded quantum dots, that is necessary for reaching the strong coupling regime. Moreover, once the right conditions are met, the reversible nature of this mechanism could permit to switch the system in and out of the strong coupling regime. Similarly, it can be used to realize photonic crystalbased switches and to modify the range of operation of photonic devices, such as add and/or drop filters or lowthreshold lasers, integrated in a 2D photonic crystal slab.

Recently, a paper was published by Mujumdar et al. ${ }^{20}$ in which measurements were reported on tip tuning of photonic crystal microcavities. These results are consistent with our results on tuning of the resonances.

The authors thank Marco Abbarchi and Lucia Cavigli for preliminary measurements. Financial support is acknowledged from the MIUR-PRIN project (Contract No. 2005025173), the European network of excellence on nanophotonics (Phoremost), the Swiss National Science Foundation (Professeur boursier program), the Swiss State Secretariate for Education and Research through COST-P11 Project No. C05-70, EU-FP6 IP "QAP” (Contract No. 15848), and the Italian MIUR-FIRB and PRIN programs.
${ }^{1}$ J. D. Joannopoulos, R. Meade, and J. Winn, Photonic Crystals (Princeton University Press, Princeton, NJ, 1995).

${ }^{2}$ T. Tanabe, M. Notomi, E. Kuramochi, A. Shinya, and H. Taniyama, Nat. Photonics 1, 49 (2007).

${ }^{3}$ H.-G. Park, S.-H. Kim, S.-H. Kwon, Y.-G. Ju, J.-K. Yang, J.-H. Baek, S.-B. Kim, and Y.-H. Lee, Science 305, 1444 (2004).

${ }^{4}$ S. Strauf, K. Hennessy, M. T. Rakher, Y.-S. Choi, A. Badolato, L. C. Andreani, E. L. Hu, P. M. Petroff, and D. Bouwmeester, Phys. Rev. Lett. 96, 127404 (2006).

${ }^{5}$ Wen-Hao Chang, Wen-Yen Chen, Hsiang-Szu Chang, Tung-Po Hsieh, Jen-Inn Chyi, and Tzu-Min Hsu, Phys. Rev. Lett. 96, 117401 (2006).

${ }^{6}$ B. S. Song, S. Noda, and T. Asano, Science 300, 1537 (2003).

${ }^{7}$ K. Hennessy, A. Badolato, M. Winger, D. Gerace, M. Atatüre, S. Gulde, S. Fält, E. L. Hu, and A. Imamogu, Nature (London) 445, 896 (2007).

${ }^{8}$ T. Yoshie, A. Scherer, J. Hendrickson, G. Khitrova, H. M. Gibbs, G. Rupper, C. Ell, O. B. Shchekin, and D. G. Deppe, Nature (London) 432, 200 (2004).

${ }^{9}$ A. Badolato, K. Hennessy, M. Atatüre, J. Dreiser, E. Hu, P. M. Petroff, and A. Imamoglum, Science 308, 1158 (2005).

${ }^{10}$ D. Dalacu, S. Frédérick, P. J. Poole, G. C. Aers, and R. L. Williams, Appl. Phys. Lett. 87, 151107 (2005).

${ }^{11}$ S. W. Leonard, J. P. Mondia, H. M. van Driel, O. Toader, S. John, K. Busch, A. Birner, U. Gösele, and V. Lehmann, Phys. Rev. B 61, R2389 (2000).

${ }^{12}$ F. Intonti, S. Vignolini, V. Türck, M. Colocci, P. Bettotti, L. Pavesi, S. L. Schweizer, R. Wehrspohn, and D. Wiersma, Appl. Phys. Lett. 89, 211117 (2006).

${ }^{13}$ S. Mosor, J. Hendrickson, B. C. Richards, J. Sweet, G. Khitrova, H. M. Gibbs, T. Yoshie, A. Scherer, O. B. Shchekin, and D. G. Deppe, Appl. Phys. Lett. 87, 141105 (2005).

${ }^{14}$ S. Strauf, M. T. Rakher, I. Carmeli, K. Hennessy, C. Meier, A. Badolato, M. J. A. DeDood, P. M. Petroff, E. L. Hu, E. G.
Gwinn, and D. Bouwmeester, Appl. Phys. Lett. 88, 043116 (2006).

${ }^{15}$ K. Hennessy, C. Högerle, E. Hu, A. Badolato, and A. Imamoglu, Appl. Phys. Lett. 89, 041118 (2006).

${ }^{16}$ A. F. Koenderink, M. Kafesaki, B. C. Buchler, and V. Sandoghdar, Phys. Rev. Lett. 95, 153904 (2005).

${ }^{17}$ I. Märki, M. Salt, and H. P. Herzig, Opt. Express 14, 2969 (2006).

${ }^{18}$ W. C. L. Hopman, K. O. van der Werf, A. J. F. Hollink, W. Bogaerts, V. Subramaniam, and R. M. de Ridder, Opt. Express 14, 8745 (2006).

${ }^{19}$ L. Lalouat, B. Cluzel, P. Velha, E. Picard, D. Peyrade, J. P. Hugonin, P. Lalanne, E. Hadji, and F. de Fornel, Phys. Rev. B 76, 041102(R) (2007).

${ }^{20}$ S. Mujumdar, A. F. Koenderink, T. Sünner, B. C. Buchler, M. Kamp, A. Forchel, and V. Sandoghdar, Opt. Express 15, 17214 (2007).

${ }^{21}$ N. Louvion, D. Gérard, J. Mouette, F. de Fornel, C. Seassal, X. Letartre, A. Rahmani, and S. Callard, Phys. Rev. Lett. 94, 113907 (2005).

${ }^{22}$ P. Kramper, M. Kafesaki, C. M. Soukoulis, A. Birner, F. Müller, U. Gösele, R. B. Wehrspohn, J. Mlynek, and V. Sandoghdar, Opt. Lett. 29, 174 (2004).

${ }^{23}$ V. S. Volkov, S. I. Bozhevolnyi, P. I. Borel, L. H. Frandsen, and M. Kristensen, Phys. Rev. B 72, 035118 (2005).

${ }^{24}$ M. Francardi, L. Balet, A. Gerardino, C. Monat, C. Zinoni, L. H. Li, B. Alloing, N. Le Thomas, R. Houdré, and A. Fiore, Phys. Status Solidi C 3, 3693 (2006).

${ }^{25}$ P. Lambelet, A. Sayah, M. Pfeffer, C. Philipona, and F. MarquisWeible, Appl. Opt. 37, 7289 (1998).

${ }^{26}$ S. Mujumdar, A. F. Koenderink, R. Wüest, and V. Sandoghdar, IEEE J. Sel. Top. Quantum Electron. 13, 253 (2007).

${ }^{27}$ C. Girard, A. Dereux, O. J. F. Martin, and M. Devel, Phys. Rev. B 50, 14467 (1994). 